\title{
Modeling and Simulation in Drug Discovery and Development
}

\section{Rahul Deshmukh*}

Pharmaceutical Sciences, College of Pharmacy, Rosalind Franklin University, USA

\section{Editorial}

The cost of healthcare is growing at a staggering rate and has taken crisis proportions. High cost of medications is one of the contributing factors to the challenge. The cost of developing new drugs is very high and also time consuming. Efforts aimed at reducing the cost and time to drug approval are of paramount importance. In addition to the traditional drug development methods, new approaches based the latest scientific knowledge and tools can help reduce both the cost and time it takes to develop and evaluate new therapies. The extensive use of computer based modeling and simulation (M\&S) can be one such valuable tool in this process. In its Critical Path Initiative, computational modeling and simulation has been identified as area that FDA expects to help speed drug development. M\&S methods are now more commonly used in drug product development process and advances in this area aimed at better prediction of in vivo outcomes can significantly impact how drugs are developed. Indeed, there are numerous examples at all stages of drug development where M\&S has enhanced decision making. M\&S can add value at various stages of the research and development process starting from the pre-clinical to the clinical stages, with continuous feedback at each stage to enhance and improve rationally guided decision making in drug discovery and development. Mechanistic biological models can separately explore the biological and compound specific parameters and integrate the information to predict absorption, distribution, metabolism and excretion (ADME), time-concentration profile of a potential drug candidate. The need to integrate large amount of data during various stages of drug development, including physiochemical characterization, in vitro assay results, formulation, animal pharmacokinetic $(\mathrm{PK})$ and pharmacodynamic (PD), toxicokinetics, coupled with commercial availability of software packages capable of handling such information has led to increased use of M\&S. Such approaches can be used to address and simulate various clinical and non-clinical outcomes. This process can help in better, faster and informed decision making leading to optimal outcomes including reduced time and cost for drug development.

Some of the questions M\&S efforts aim to address are - what is the likely clinical PK profile of the drug candidate, what should be the optimal dosing schedule and what would be an appropriate route of administration, what will be the extent of population variability and how will it impact the safety and efficacy of the candidate, etc. As mentioned, M\&S is being applied to all stages of drug development. One such area has been its application prediction of absorption and the pharmacokinetics of a drug. Over the past decade there has been intensive research to understand the behavior of drugs in vivo based on their physiochemical properties. The better understanding of the physiochemical properties, along with improved knowledge of the physiological conditions in the gastrointestinal tract, the availability of in vitro and in vivo animal PK data, has led to development of better in silico model to study oral drug absorption and distribution. Physiological based pharmacokinetic (PBPK) models aim to use physiological and species-specific parameters including blood flow to tissues, size of a tissue, tissue partitioning, enzyme abundance, etc along with compound specific parameters o describe the ADME processes. $\mathrm{PK} / \mathrm{PD}$ models can be incorporated early into drug discovery to integrate knowledge of drug action and disease progression, describe experimental data and also predict outcomes under different dosing schedules, routes of administration, better design of clinical trials. As more in vitro and in vivo data is generated, and models undergo continuous refinement, simulations can also help to support safety assessment, study effect of formulations and evaluate potential of drugdrug interactions.

In comparison to more widely used empirical approaches such as non-compartmental analysis which require prior information on in vivo time-concentration profile to describe PK parameters, PBPK modeling can start at an very early stage where combination of knowledge of physiology, physiochemical and biochemical properties, in vitro screening data of the drug can be used to simulate the pharmacokinetic profile based on certain assumptions. This process can be iterative and as in vivo data gets generated the PBPK model can be refined to incorporate the new information. Furthermore, such models provide a framework for incorporating physiological and biochemical differences across species, allowing for more rational approaches to interspecies scaling. At the lead discovery stage, using the in vitro data, M\&S can guide the design of the screening cascade and identify compounds that are likely to have good PK properties before animal testing. Compound advancing further in to human studies, can be modeled to predict the human PK and dose required for efficacy without having adverse side effects. Through M\&S, the first in human studies can be supported with optimal clinical trial design to capture population variability, prediction of food effects and design of more focused trial to obtain critical information such as inclusion/exclusion of population based on pharmacogenomics, etc. During clinical development, M\&S can aid in refinement clinical trial design, modeling PK/PD in special population such as renal and hepatic dysfunction, pediatrics, disease population, etc. M\&S also has great value to test hypotheses and potential scenarios that may be encountered during the drug development process. For example, if in vitro data suggests that the drug candidate is an inhibitor of drug metabolizing enzyme or metabolized by a specific enzyme, virtual PBPK bases trails can be explored to test the impact of enzyme interaction/ inhibition on concentration-time profile. This in turn can help design better clinical trial to address drug-drug interaction issues that may arise during clinical development.

*Corresponding author: Rahul Deshmukh, Pharmaceutical Sciences, College of Pharmacy, Rosalind Franklin University, USA, Tel: 847-578-3233; E-mail: Rahul.Deshmukh@rosalindfranklin.edu

Received September 26, 2012; Accepted September 27, 2012; Published September 30, 2012

Citation: Deshmukh R (2012) Modeling and Simulation in Drug Discovery and Development. J Bioequiv Availab 4: xxvii-xxviii. doi:10.4172/jbb.10000e22

Copyright: $@ 2012$ Deshmukh R. This is an open-access article distributed under the terms of the Creative Commons Attribution License, which permits unrestricted use, distribution, and reproduction in any medium, provided the original author and source are credited. 
Citation: Deshmukh R (2012) Modeling and Simulation in Drug Discovery and Development. J Bioequiv Availab 4: xxvii-xxviii. doi:10.4172/ jbb.10000e22

M\&S makes itself very amenable to the "learn-and-confirm" paradigm that has been proposed as an efficient and rational approach to drug development. By timely application of M\&S at each stage of drug development it is possible to effectively implement this paradigm. Despite its promise, M\&S do have limitations and weaknesses. They are based on our current understanding of the biological process which may not be completely understood leading to information gaps. Researchers need to have adequate background knowledge of the biochemical and biological process to understand the limitation and assumptions incorporated in the models to accurately interpret and judge the results. For this approach to gain wide acceptance, prospective examples that assure the clinical value of such a modeling technique are needed. In the coming years, modeling and simulation based approaches will keep evolving and become mainstream. The predictive power of such approaches can be harnessed to prospectively answer critical question in the drug development efforts, eventually leading to faster, safer and more economical drug development efforts. 